\title{
Accelerated nutrition transition in the North West Province of South Africa: results from the Prospective Urban and Rural Epidemiology (PURE-NWP-SA) cohort study, 2005 to 2010
}

\author{
Edelweiss Wentzel-Vilioen ${ }^{1, *}$, Sarie Lee ${ }^{1}$, Ria Laubscher ${ }^{2}$ and Hester H Vorster ${ }^{1}$ \\ ${ }^{1}$ Centre of Excellence for Nutrition, Faculty of Health Sciences, North-West University, Private Bag X6003, \\ Potchefstroom, South Africa: ${ }^{2}$ South African Medical Research Council, Biostatistics Unit, Tygerberg, South Africa
}

Submitted 15 October 2017: Final revision received 14 March 2018: Accepted 26 March 2018: First published online 8 May 2018

\begin{abstract}
Objective: South Africa (SA) is in the midst of a health transition characterized by a quadruple burden of diseases and a nutrition transition. The existing nutrition transition in SA, accompanied by the coexistence of under- and overnutrition in the population, motivated the present study. Its objectives were to measure and report the changes in nutrient intakes of rural and urban black Africans over time to assess the impact of urbanization and modernization of lifestyles on dietary intakes and non-communicable disease (NCD) risk.

Design: The PURE-NWP-SA study recruited 2000 black South African volunteers aged 35-70 years in 2005, of which detailed nutrient intakes from 1858 participants were available. In 2010 nutrient intakes of a cohort of 1154 participants were measured.

Results: Median energy intake increased over time. In 2010, rural participants consumed the amount of energy (men $9.7 \mathrm{MJ} / \mathrm{d}$; women $9.1 \mathrm{MJ} / \mathrm{d}$ ) that urban participants consumed in 2005 (men $9.9 \mathrm{MJ} / \mathrm{d}$; women $9.0 \mathrm{MJ} / \mathrm{d}$ ). The nutrition transition was characterized by increases in the percentage of energy from animal protein, total fat (rural men and women), saturated (not urban women) and monounsaturated fat, as well as added sugar. Despite the higher energy intake, not all the participants met total micronutrient needs in 2010.

Conclusions: The PURE nutrient intake data confirmed that the nutrition transition in the North West Province of SA is extremely rapid in rural areas. The shift towards higher energy intakes, an animal food-based diet, higher intakes of fat and lower intake of fibre, at the cost of lower plant protein and starchy food intakes, could increase the risk of NCD.
\end{abstract}

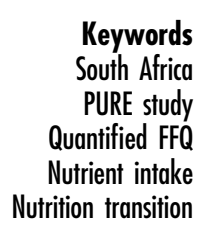

It is recognized that South Africa (SA), a low-middle-income country, is in the midst of a health transition characterized by a quadruple burden of communicable diseases, noncommunicable diseases (NCD), perinatal and maternal and injury-related disorders ${ }^{(1)}$, as well as experiencing a nutrition transition ${ }^{(2)}$. Sharp increases in overweight, obesity, hypertension, diabetes and CVD, without substantial improvements in undernutrition among children, have been reported for all population groups ${ }^{(3-5)}$. The South African Medical Research Council ${ }^{(6)}$ identified both underand overnutrition as risk factors for disability-adjusted life years and mortality in the South African population. We have previously shown that the diet followed by black Africans in rural areas of the North West Province (NWP) does not meet micronutrient requirements ${ }^{(7-10)}$. However, with urbanization and 'modernization' of the diet, increases (but not optimization) of micronutrient intakes have been observed $^{(7)}$. Simultaneously, macronutrient intakes have been characterized by increases in animal protein, total fat and especially added sugar ${ }^{(7,11)}$ while total carbohydrate intake has decreased ${ }^{(7)}$, despite increased intake of added sugar ${ }^{(11)}$. These changes in nutrient intakes have been described since the $1970 \mathrm{~s}^{(2)}$. To address micronutrient deficiencies, the South African Department of Health (Directorate Nutrition) instituted a mandatory micronutrient fortification of the staples, maize meal and bread flour, in $2003^{(12)}$ that came into effect in 2004 . The objectives of the present study were to measure and report the changes in nutrient intakes of rural and urban black African men and women over time (from 2005 to 2010) to assess the impact of urbanization and modernization of lifestyles on dietary and therefore nutrient intakes and NCD risk. 


\section{Participants and methods}

\section{The PURE study}

The Prospective Urban and Rural Epidemiology (PURE) study is a prospective cohort study tracking changing lifestyles, risk factors and chronic disease in urban and rural areas of seventeen countries in transition ${ }^{(13,14)}$. One of the legs of the PURE study in SA is being conducted in two areas (urban and rural) of the NWP (PURE-NWP-SA). The province is experiencing a typical epidemiological transition ${ }^{(7)}$. The baseline study was conducted in 2005 and the follow-up in 2010.

The design of the PURE-NWP-SA study has been described elsewhere ${ }^{(11,14,15)}$. Briefly, participants were recruited from four different sites (two rural and two urban) in the NWP of SA. A census profile of 6000 households (1500 in each area) was completed from which 4000 individuals were identified as meeting the inclusion criteria and visited at home. The study was explained to the individuals and after voluntary and informed consent was obtained, the questionnaires were completed. Questionnaires for 3750 participants were completed. These participants were invited to the study sites and after giving informed consent, blood samples were drawn and other measurements taken. The dietary questionnaires were also completed at this stage. In total, 2000 participants were tested (target of 500 in each community) in 2005. In the 5-year follow-up survey conducted in 2010, 1233 participants were included. Figure 1 provides a summary of the recruitment and follow-up procedures (adapted from Vorster et al. ${ }^{(11)}$ ).

\section{Measurements}

\section{Questionnaires}

Standardized structured demographic, socio-economic, lifestyle and physical activity questionnaires of the

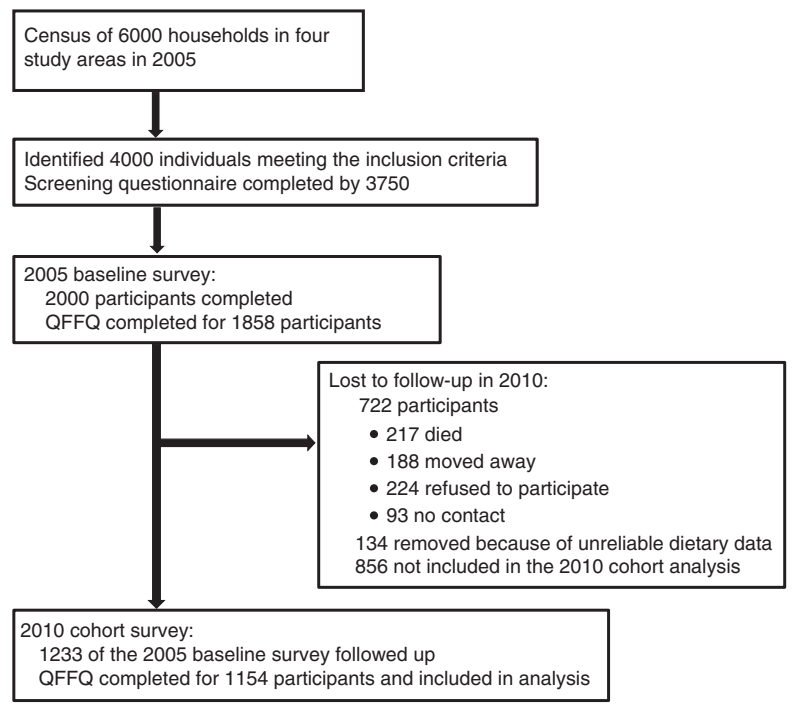

Fig. 1 Participant recruitment and follow-up from years 2005 to 2010 (adapted from Vorster et al. ${ }^{(11)}$ ). QFFQ, quantified FFQ international PURE study were used ${ }^{(13)}$. These questionnaires were adapted for the South African study where necessary. Sixteen volunteer fieldworkers, from the communities where the study was undertaken, were recruited and intensively trained to complete the questionnaires at the study site or participant's home in the language of choice (Setswana, Afrikaans or English). A 145-item, culture-sensitive, quantified FFQ (QFFQ) was used to obtain dietary intakes covering the previous month. The QFFQ was previously developed and extensively validated for this population ${ }^{(16,17)}$. Reproducibility was tested again for the present study ${ }^{(18)}$. Portion sizes were reported by the participants using a food-portion photograph book ${ }^{(19)}$ specifically developed and tested for this population, as well as other suitable tools as previously described ${ }^{(18)}$. Portion sizes reported in grams (based on the photographic portion book) or household measures were converted to weights using standard tables ${ }^{(20)}$. Nutrient intake was calculated using the South African food composition database ${ }^{(21)}$. Food items not in the database were either analysed or sourced from other databases, added to the database and then coded as previously described ${ }^{(18)}$.

\section{Other measurements}

The methodology used for the anthropometric measures (height, weight and waist circumference), blood collection, biochemical analyses (HDL-cholesterol) and HIV testing have been described previously ${ }^{(11,14)}$.

\section{Statistical analyses}

The statistical software package Stata version 14 was used for the analyses. Due to the skewness of the nutrient intake data, results are reported as median and 25th percentile-75th percentile. Wilcoxon's two-sample test was used to test for differences between the rural and urban participants (by gender) in terms of their nutrient intakes, as well as the changes in nutrient intakes between 2005 and 2010. Wilcoxon's signed-rank test used to test for changes in nutrient intakes between 2005 and 2010 for each of the four groups (by gender and study site). Participants with energy intakes $\geq 30000$ or $\leq 3000 \mathrm{~kJ} / \mathrm{d}$ in either 2005 or 2010 were excluded from the data set ${ }^{(11)}$.

To evaluate adequacy of intakes as well as compliance to recommendations for the prevention of NCD, reported nutrient intakes were compared with international recommendations $^{(22)}$, WHO recommendations ${ }^{(23)}$ as well as the South African food-based dietary guidelines ${ }^{(24)}$, as shown in the online supplementary material, Supplemental Table 1.

Baseline mean characteristics of the cohort in 2005 and of those participants lost to follow-up in 2010 are shown in Table 1.

In addition to intakes reported in their respective units, intakes of macronutrients were also expressed as a percentage of total energy, and intakes of micronutrients and dietary fibre per $4.2 \mathrm{MJ} \quad(1000 \mathrm{kcal}$; see online 
Table 1 Baseline characteristics in 2005 of participants followed up from 2005 to 2010 (the cohort), compared with subjects lost to follow-up and compared with 2010; PURE-NWP-SA study

\begin{tabular}{|c|c|c|c|c|c|c|c|c|c|c|c|}
\hline \multirow[b]{2}{*}{ Variable } & \multicolumn{3}{|c|}{$\begin{array}{l}\text { Baseline data in } 2005 \text { of } \\
\text { participants followed up } \\
\text { in } 2010\end{array}$} & \multicolumn{3}{|c|}{$\begin{array}{l}\text { Baseline data in } 2005 \text { of } \\
\text { subjects lost to follow-up } \\
\text { in } 2010\end{array}$} & \multirow[b]{2}{*}{$P$ value ${ }^{*}$} & \multicolumn{3}{|c|}{$\begin{array}{l}\text { Cohort data of participants } \\
\text { in } 2010\end{array}$} & \multirow[b]{2}{*}{$P$ valuet } \\
\hline & $n$ & Mean & SD & $n$ & Mean & SD & & $n$ & Mean & SD & \\
\hline Age (years) & 1154 & $50 \cdot 8$ & $10 \cdot 3$ & 856 & 48.6 & $10 \cdot 4$ & $<0.001$ & 1154 & - & & - \\
\hline Sex (male; \%) & 1154 & \multicolumn{2}{|r|}{33.6} & 856 & \multicolumn{2}{|r|}{41.8} & $<0.001$ & 1154 & - & & - \\
\hline Locality (rural; \%) & 1154 & \multicolumn{2}{|r|}{$51 \cdot 7$} & 856 & \multicolumn{2}{|r|}{47.8} & 0.080 & 1154 & - & & - \\
\hline $\begin{array}{l}\text { Education attainment } \\
\text { (no formal education; \%) }\end{array}$ & 1154 & \multicolumn{2}{|r|}{$36 \cdot 0$} & 856 & \multicolumn{2}{|r|}{$32 \cdot 1$} & 0.073 & 1154 & - & & - \\
\hline HIV positive (\%) & 1154 & \multicolumn{2}{|r|}{$17 \cdot 1$} & 856 & \multicolumn{2}{|r|}{$22 \cdot 2$} & 0.004 & 1154 & $4.9 \% r$ & ew cases & - \\
\hline BMI $\left(\mathrm{kg} / \mathrm{m}^{2}\right) \ddagger$ & 1154 & $25 \cdot 1$ & 7.0 & 853 & $24 \cdot 1$ & 7.0 & $<0.001$ & 1154 & $25 \cdot 5$ & $7 \cdot 3$ & $<0.001$ \\
\hline Waist circumference $(\mathrm{cm}) \ddagger$ & 1147 & $80 \cdot 3$ & $12 \cdot 8$ & 843 & $79 \cdot 0$ & $13 \cdot 3$ & 0.007 & 1147 & 81.9 & 13.0 & $<0.001$ \\
\hline \multirow[t]{2}{*}{ HDL-cholesterol $(\mathrm{mmol} / \mathrm{l}) \ddagger$} & 1087 & 1.54 & 0.61 & 805 & 1.48 & 0.66 & 0.006 & 1087 & 1.42 & 0.59 & $<0.001$ \\
\hline & $n$ & Median & P25-P75 & $n$ & Median & P25-P75 & $P$ value* & $n$ & Median & P25-P75 & $P$ value $\dagger$ \\
\hline Energy intake (MJ/d) & 1154 & 7.4 & $5 \cdot 6-10 \cdot 1$ & 796 & 6.9 & $4 \cdot 7-9 \cdot 8$ & 0.003 & 1154 & $10 \cdot 5$ & $7 \cdot 5-13.9$ & $<0.0001$ \\
\hline
\end{tabular}

PURE-NWP-SA, Prospective Urban and Rural Epidemiology in the North West Province of South Africa; P25, 25th percentile; P75, 75th percentile. ${ }^{*} P$ value for significance of differences between subjects followed up in 2010 and those lost to follow-up (categorical variables: $X^{2}$ test; continuous variables: twosample Wilcoxon test).

$\dagger P$ value for significance of differences between the cohort in 2005 and 2010 (categorical variables: $x^{2}$ test; continuous variables: two-sample Wilcoxon test).

supplementary material, Supplemental Table 2; Tables 2 and 3). The percentages of participants not reaching $100 \%$ of recommended intakes were also calculated and are shown in Tables 4 and 5 .

\section{Results}

\section{Comparison of the cobort with those lost from the} study and between 2005 and 2010

Dietary intake data of 1858 participants were measured in 2005. Table 1 shows the characteristics (in 2005) of the 1154 cohort participants who could be followed up in 2010 compared with the baseline characteristics of the 856 participants lost to follow-up. The latter were slightly (but significantly) younger, with a higher proportion of men, more HIV-infected participants, with lower BMI, waist circumference, HDL-cholesterol and total energy intake. There were small (but significant) increases in BMI and waist circumference from 2005 to 2010 that could probably be explained by the increase in energy intake and ageing.

\section{Nutrient intakes, macronutrient distribution and micronutrient density of all participants in 2005}

Supplemental Table 2 (see online supplementary material) shows that in 2005 the nutrient intakes of urban men and women were consistently higher than those of their rural counterparts.

\section{Changes in nutrient intakes of men from 2005 to 2010}

In Table 2, nutrient intakes of the male cohort (186 rural men and 202 urban men) in 2005 are compared with the intakes in 2010. Rural men consumed significantly more energy and therefore significantly more of all nutrients
$(P<0.05$; except Mn) in 2010 than in 2005. However, the percentage of energy contributed by plant protein and the contribution of total carbohydrate to energy intake were lower in 2010 than in 2005, while the energy contributions from total and animal protein, total fat, saturated fat, monounsaturated fat and added sugar were higher in 2010 than in 2005. Energy from alcohol decreased significantly from 2005 to 2010 . There were also significant increases in some micronutrient intakes (Fe, niacin and vitamin $\mathrm{C}$ ), even when these micronutrients were expressed in terms of energy intake to 'correct' for increased energy intakes in 2010.

Urban men also showed a significant increase in total energy intake from 2005 to $2010(P<0 \cdot 001)$ and therefore an increased intake of all other nutrients. A non-significant decrease in the percentage of energy from alcohol was measured. No change in the percentage contribution of total protein to energy intake was observed, but similar to rural men, significant increases in the percentage contributions of animal protein, saturated and monounsaturated fats, as well as added sugar, to energy were seen. The percentage of energy from plant protein and total carbohydrate decreased significantly in rural and urban men from 2005 to 2010. Significant increases in micronutrient density for niacin and vitamin $\mathrm{C}$ and significant decreases for fibre and $\mathrm{Zn}$ were measured.

\section{Changes in nutrient intakes of women from 2005 to 2010}

Table 3 compares the changes in nutrient intakes over time in the female cohort ( 411 rural women and 355 urban women). Both rural and urban women consumed more energy in 2010 compared with 2005, with the consequence that intakes of all nutrients were higher in 2010 in the respective groups. However, as in the case of men, 
Table 2 Daily nutrient intake, energy distribution and nutrient density of the male cohort by rural/urban residence in 2005 and 2010 ; PURE-NWP-SA study

\begin{tabular}{|c|c|c|c|c|c|c|c|c|c|c|c|}
\hline \multirow[b]{3}{*}{ Energy and nutrients } & \multicolumn{4}{|c|}{ Rural men ( $n$ 186) } & \multirow[b]{3}{*}{$P$ value* } & \multicolumn{4}{|c|}{ Urban men (n 202) } & \multirow[b]{3}{*}{$P$ value ${ }^{*}$} & \multirow[b]{3}{*}{$P$ valuet } \\
\hline & \multicolumn{2}{|r|}{2005} & \multicolumn{2}{|c|}{2010} & & \multicolumn{2}{|r|}{2005} & \multicolumn{2}{|c|}{2010} & & \\
\hline & Median & P25-P75 & Median & P25-P75 & & Median & P25-P75 & Median & P25-P75 & & \\
\hline Energy (MJ) & 6.9 & $5 \cdot 6-8 \cdot 8$ & $9 \cdot 7$ & $6.95-13.8$ & $<0.001$ & 9.9 & $7 \cdot 2-12 \cdot 6$ & $13 \cdot 7$ & $10.5-17.89$ & $<0.001$ & 0.035 \\
\hline Total protein (g) & 44.4 & $35 \cdot 3-61 \cdot 2$ & $66 \cdot 2$ & $46 \cdot 79-91.5$ & $<0.001$ & $72 \cdot 8$ & $52.5-95.8$ & $100 \cdot 0$ & $75 \cdot 0-138 \cdot 4$ & $<0.001$ & 0.042 \\
\hline Plant protein (g) & $30 \cdot 4$ & $22 \cdot 2-39 \cdot 7$ & 33.8 & $24.4-51.5$ & 0.008 & $36 \cdot 9$ & $27 \cdot 6-47 \cdot 3$ & 43.5 & $32 \cdot 9-63 \cdot 5$ & $<0.001$ & 0.087 \\
\hline Animal protein (g) & $12 \cdot 6$ & $8 \cdot 0-19.5$ & $25 \cdot 8$ & $16 \cdot 4-45 \cdot 2$ & $<0.001$ & $30 \cdot 0$ & $20 \cdot 8-43.5$ & $51 \cdot 0$ & $37 \cdot 1-75 \cdot 3$ & $<0.001$ & 0.058 \\
\hline Total fat $(\mathrm{g})$ & $31 \cdot 2$ & $24 \cdot 8-43 \cdot 1$ & $54 \cdot 6$ & $36 \cdot 4-79.4$ & $<0.001$ & 61.6 & $44.4-86 \cdot 2$ & 94.4 & $65.9-131.9$ & $<0.001$ & 0.337 \\
\hline Saturated fat $(\mathrm{g})$ & 6.9 & $4 \cdot 8-9 \cdot 7$ & $15 \cdot 6$ & $10 \cdot 2-23 \cdot 5$ & $<0.001$ & $15 \cdot 1$ & $10 \cdot 6-21 \cdot 5$ & 24.9 & $17 \cdot 3-35 \cdot 4$ & $<0.001$ & 0.877 \\
\hline Monounsaturated fat (g) & $7 \cdot 3$ & $4.7-10.7$ & $15 \cdot 5$ & $9 \cdot 6-22 \cdot 9$ & $<0.001$ & $16 \cdot 7$ & $11.99-24.8$ & $28 \cdot 1$ & $20 \cdot 6-40 \cdot 7$ & $<0.001$ & 0.392 \\
\hline Polyunsaturated fat (g) & $10 \cdot 3$ & $7 \cdot 3-14 \cdot 3$ & $16 \cdot 3$ & $9 \cdot 99-26 \cdot 3$ & $<0.001$ & $18 \cdot 0$ & $12 \cdot 5-25 \cdot 7$ & $25 \cdot 6$ & $16 \cdot 8-39 \cdot 2$ & $<0.001$ & 0.694 \\
\hline Cholesterol (mg) & 111 & $72-171$ & 210 & 87-377 & $<0.001$ & 249 & 163-355 & 408 & $279-657$ & $<0.001$ & 0.027 \\
\hline Total carbohydrate (g) & 257.5 & $199 \cdot 0-334.96$ & 333.4 & $231 \cdot 6-458.6$ & $<0.001$ & 330.8 & $238.6-431.6$ & 419.4 & $306 \cdot 7-584 \cdot 2$ & $<0.001$ & 0.100 \\
\hline Added sugar (g) & 23.9 & $14.27-33.06$ & $45 \cdot 3$ & $23.9-82.5$ & $<0.001$ & 34.7 & $20 \cdot 9-56 \cdot 1$ & 59.3 & $36.5-99.7$ & $<0.001$ & 0.365 \\
\hline Dietary fibre $(\mathrm{g})$ & 18.5 & $14 \cdot 2-25 \cdot 2$ & $20 \cdot 6$ & $14 \cdot 2-31 \cdot 1$ & 0.003 & $27 \cdot 3$ & $17.5-35.4$ & 31.4 & $23 \cdot 1-43 \cdot 1$ & $<0.001$ & 0.066 \\
\hline $\mathrm{Ca}(\mathrm{mg})$ & 229 & $156-345$ & 339 & $192-552$ & $<0.001$ & 397 & $292-573$ & 596 & $432-800$ & $<0.001$ & 0.073 \\
\hline $\mathrm{Fe}(\mathrm{mg})$ & $12 \cdot 2$ & $9 \cdot 1-15 \cdot 9$ & $16 \cdot 0$ & $11 \cdot 4-23 \cdot 1$ & $<0.001$ & $16 \cdot 0$ & $11 \cdot 2-21.9$ & 22.2 & $16 \cdot 2-30 \cdot 1$ & $<0.001$ & 0.012 \\
\hline $\mathrm{Mg}(\mathrm{mg})$ & 297 & $214-452$ & 402 & $285-81$ & $<0.001$ & 389 & $288-539$ & 493 & $369-745$ & $<0.001$ & 0.208 \\
\hline$P(\mathrm{mg})$ & 847 & $646-1113$ & 1206 & 839-1638 & $<0.001$ & 1107 & $856-1477 \cdot 2$ & 1642 & 1166-2229 & $<0.001$ & 0.011 \\
\hline $\mathrm{K}(\mathrm{mg})$ & 1394 & $1089-1759$ & 2441 & $1711-3243$ & $<0.001$ & 2078 & $1526-2809$ & 3326 & $2420-4378$ & $<0.001$ & 0.267 \\
\hline $\mathrm{Zn}(\mathrm{mg})$ & 8.6 & $6 \cdot 7-11 \cdot 3$ & $13 \cdot 3$ & $9 \cdot 5-20 \cdot 2$ & $<0.001$ & 12.5 & $9 \cdot 0-17$ & 18.4 & $14 \cdot 1-26 \cdot 5$ & $<0.001$ & 0.038 \\
\hline $\mathrm{Cu}(\mathrm{mg})$ & $1 \cdot 1$ & $0.8-1.5$ & 1.4 & $0.9-1.9$ & $<0.001$ & 1.5 & $1 \cdot 1-1.9$ & 1.9 & $1 \cdot 4-2 \cdot 6$ & $<0.001$ & 0.019 \\
\hline $\mathrm{Mn}(\mu \mathrm{g})$ & 1749 & 1110-3259 & 2061 & $1329-3124$ & 0.690 & 2647 & $1899-3866$ & 3132 & $2219-4188$ & 0.008 & 0.156 \\
\hline Vitamin A ( $\mu \mathrm{g})$ & 4267 & $274-712$ & 941 & $535-1727$ & $<0.001$ & 890 & $536-1447$ & 1742 & $1042-2572$ & $<0.001$ & 0.019 \\
\hline Thiamin (mg) & 1.6 & $1 \cdot 2-2 \cdot 1$ & 1.9 & $1 \cdot 3-2 \cdot 8$ & $<0.001$ & 1.9 & $1 \cdot 3-2 \cdot 7$ & 2.5 & $1 \cdot 8-3 \cdot 7$ & $<0.001$ & 0.030 \\
\hline Riboflavin (mg) & 0.9 & $0.7-1.4$ & 1.4 & $0.97-1.97$ & $<0.001$ & 1.5 & $1 \cdot 1-2 \cdot 1$ & $2 \cdot 3$ & $1.5-3.1$ & $<0.001$ & $<0.001$ \\
\hline $\operatorname{Niacin}(\mathrm{mg})$ & $12 \cdot 2$ & $9 \cdot 4-17 \cdot 6$ & $24 \cdot 1$ & $17 \cdot 0-34.8$ & $<0.001$ & 18.5 & $14 \cdot 0-25 \cdot 1$ & 34.4 & $25 \cdot 2-49 \cdot 7$ & $<0.001$ & $<0.001$ \\
\hline Vitamin $B_{6}(\mathrm{mg})$ & $1 \cdot 2$ & $0.94-1.6$ & 2.9 & $1.9-4.3$ & $<0.001$ & $1 \cdot 8$ & $1 \cdot 2-2 \cdot 7$ & 4.4 & $3 \cdot 0-6 \cdot 4$ & $<0.001$ & $<0.001$ \\
\hline Folate $(\mu \mathrm{g})$ & 356.9 & $268.9-467.5$ & $467 \cdot 7$ & $300 \cdot 8-744.9$ & $<0.001$ & 443.0 & $307 \cdot 3-626 \cdot 5$ & 675.9 & $485 \cdot 3-1022 \cdot 8$ & $<0.001$ & 0.003 \\
\hline Vitamin $B_{12}(\mu \mathrm{g})$ & 1.8 & $0.9-2.95$ & 2.73 & $1.3-5.5$ & $<0.001$ & 4.8 & $2 \cdot 4-7.7$ & 6.4 & $3 \cdot 8-11.9$ & $<0.001$ & 0.136 \\
\hline Pantothenic acid (mg) & 2.9 & $2 \cdot 2-4 \cdot 1$ & $4 \cdot 6$ & $3 \cdot 1-6.5$ & $<0.001$ & 4.9 & $3 \cdot 6-6 \cdot 6$ & 8.61 & $6 \cdot 2-11 \cdot 4$ & $<0.001$ & $<0.001$ \\
\hline Biotin $(\mu \mathrm{g})$ & $25 \cdot 0$ & $18 \cdot 2-31 \cdot 9$ & $30 \cdot 8$ & $19 \cdot 2-48 \cdot 4$ & $<0.001$ & $45 \cdot 8$ & $29 \cdot 5-61 \cdot 7$ & 62.5 & $41.9-88.7$ & $<0.001$ & 0.025 \\
\hline Vitamin C (mg) & 12 & $7-16$ & 29 & $14-56$ & $<0.001$ & 33 & $20-57$ & 62 & $36-97$ & $<0.001$ & 0.447 \\
\hline Vitamin D (mg) & $1 \cdot 7$ & $0.9-2.7$ & 2.4 & $0.96-5.1$ & $<0.001$ & 3.2 & $1.98-4.8$ & $5 \cdot 0$ & $2 \cdot 8-8 \cdot 2$ & $<0.001$ & 0.040 \\
\hline Vitamin E (mg) & 8.4 & $5 \cdot 2-11 \cdot 9$ & 11.9 & $6 \cdot 1-19 \cdot 6$ & $<0.001$ & $12 \cdot 4$ & $8 \cdot 3-17 \cdot 4$ & $16 \cdot 0$ & $10 \cdot 9-24 \cdot 2$ & $<0.001$ & 0.742 \\
\hline Alcohol (g) & 4.3 & $0-32$ & 0.7 & $0-18 \cdot 8$ & 0.012 & 11.4 & $0-27 \cdot 7$ & $7 \cdot 1$ & $0-26.5$ & 0.587 & 0.187 \\
\hline \multicolumn{12}{|l|}{ Energy distribution } \\
\hline$\%$ of TE from protein & $10 \cdot 6$ & $9 \cdot 8-11 \cdot 6$ & $11 \cdot 3$ & $9 \cdot 5-13.5$ & 0.005 & $12 \cdot 5$ & $11 \cdot 6-13.5$ & $12 \cdot 7$ & $11 \cdot 3-14 \cdot 3$ & 0.483 & 0.088 \\
\hline$\%$ of TE from animal protein & 3.1 & $2 \cdot 0-4 \cdot 2$ & 4.6 & $2.9-7.5$ & $<0.001$ & 5.4 & $4.3-6.9$ & $6 \cdot 8$ & $5 \cdot 1-8.5$ & $<0.001$ & 0.109 \\
\hline$\%$ of TE from plant protein & 7.5 & $6 \cdot 7-8 \cdot 1$ & $6 \cdot 2$ & $5 \cdot 2-7 \cdot 2$ & $<0.001$ & $6 \cdot 5$ & $5 \cdot 7-7 \cdot 2$ & $5 \cdot 7$ & $4 \cdot 8-6 \cdot 3$ & $<0.001$ & 0.014 \\
\hline$\%$ of TE from total fat & $17 \cdot 8$ & $13 \cdot 6-23 \cdot 1$ & 21.5 & $15 \cdot 7-28.9$ & $<0.001$ & 24.9 & $21 \cdot 6-29 \cdot 4$ & $26 \cdot 3$ & $22 \cdot 1-31 \cdot 3$ & 0.329 & 0.003 \\
\hline$\%$ of TE from saturated fat & 3.9 & $2 \cdot 5-5 \cdot 1$ & $6 \cdot 2$ & $4.1-8.5$ & $<0.001$ & 6.4 & $5 \cdot 1-7 \cdot 6$ & $6 \cdot 9$ & $5 \cdot 7-8 \cdot 8$ & 0.001 & $<0.001$ \\
\hline$\%$ of TE from monounsaturated fat & 3.9 & $2.7-5.5$ & $6 \cdot \overline{3}$ & $4.3-8 \cdot 6$ & $<0.001$ & $7 \cdot 1$ & $5 \cdot 8-8.4$ & 8.0 & $6 \cdot 5-9 \cdot 8$ & 0.001 & $<0.001$ \\
\hline$\%$ of TE from polyunsaturated fat & 5.9 & $4 \cdot 1-7.9$ & $6 \cdot 1$ & $4 \cdot 2-8.9$ & 0.021 & $7 \cdot 2$ & $5 \cdot 7-8 \cdot 8$ & $7 \cdot 2$ & $5 \cdot 7-9 \cdot 1$ & 0.943 & 0.106 \\
\hline$\%$ of TE from total carbohydrate & 63.8 & $58 \cdot 1-69 \cdot 7$ & 59.5 & $51 \cdot 6-66.5$ & $<0.001$ & $56 \cdot 1$ & $52 \cdot 0-60 \cdot 6$ & $54 \cdot 3$ & $48 \cdot 4-59 \cdot 7$ & 0.011 & 0.113 \\
\hline$\%$ of TE from added sugar & $5 \cdot 8$ & $2.9-8.3$ & $8 \cdot 1$ & $4.5-14.2$ & $<0.001$ & 6.5 & $4 \cdot 5-9 \cdot 2$ & 7.9 & $4 \cdot 5-12 \cdot 0$ & 0.001 & 0.048 \\
\hline$\%$ of TE from alcohol & $2 \cdot 0$ & $0-12 \cdot 5$ & 0.3 & $0-6.0$ & $<0.001$ & 3.5 & $0-7.9$ & 1.4 & $0-4.9$ & 0.012 & 0.204 \\
\hline
\end{tabular}


the changes in macronutrient distribution and micronutrient density showed distinct patterns.

In rural women, significant increases in the percentage of energy from animal protein, total, saturated, monounsaturated and polyunsaturated fats, as well as added sugar, were observed. Significant decreases in the percentage of energy from plant protein and total carbohydrate were seen. Furthermore, significant improvements in the nutrient density of $\mathrm{Ca}, \mathrm{Fe}$, niacin and vitamin $\mathrm{C}$ occurred, but the density of dietary fibre and thiamin decreased.

In urban women, percentage of energy from animal protein increased, while that from plant protein decreased. The change in percentage of energy from total fat was not significant. There was a significant decrease in the energy contribution from total carbohydrate between 2005 and 2010, but a significant increase from added sugar. Significant improvements in the nutrient density of $\mathrm{Fe}$, niacin and vitamin $\mathrm{C}$ were seen, while that of $\mathrm{Zn}$ decreased.

\section{Comparison of the differences in change of nutrient intakes between rural and urban participants}

A possible question is whether the changes in nutrient intakes from 2005 to 2010 were significantly greater or smaller in rural than in urban participants. We therefore calculated the significance of the effect of time, shown in the final column of Tables 2 and 3.

\section{Differences in change of nutrient intakes from 2005 to 2010 between rural and urban men}

The significance of the differences in the change of nutrient intakes from 2005 to 2010 between rural and urban men is shown in Table 2. Because the change in energy intake from 2005 to 2010 was $1172 \mathrm{~kJ} / \mathrm{d}$ greater in urban men, the changes in intakes of total protein, cholesterol and most micronutrients were also greater in urban men. However, when macronutrient intakes are expressed as a percentage of total energy and micronutrients intakes are expressed per $4.2 \mathrm{MJ}$, the changes in rural men were actually larger than those in urban men. These differences were significant for percentage of energy from plant protein, total, saturated and monounsaturated fats, and added sugar. The change in nutrient density was significantly higher in the rural men compared with the urban men for Fe.

\section{Differences in change of nutrient intakes from 2005 to 2010 between rural and urban women}

Table 3 shows that the median change in energy from 2005 to 2010 was slightly more in rural women $(2 \cdot 6 v$. $2 \cdot 3 \mathrm{MJ} / \mathrm{d})$ than urban women $(P=0 \cdot 049)$. There were also other significant differences, for example the change in total fat intake was significantly greater in rural women. 
Table 3 Daily nutrient intake, energy distribution and nutrient density of the female cohort by rural/urban residence in 2005 and 2010 ; PURE-NWP-SA study

\begin{tabular}{|c|c|c|c|c|c|c|c|c|c|c|c|}
\hline \multirow[b]{3}{*}{ Energy and nutrients } & \multicolumn{4}{|c|}{ Rural women ( $n$ 411) } & \multirow[b]{3}{*}{$P \dagger$} & \multicolumn{4}{|c|}{ Urban women ( $n$ 355) } & \multirow[b]{3}{*}{$P$ value* } & \multirow[b]{3}{*}{$P$ value } \\
\hline & \multicolumn{2}{|c|}{2005} & \multicolumn{2}{|c|}{2010} & & \multicolumn{2}{|c|}{2005} & \multicolumn{2}{|c|}{2010} & & \\
\hline & Median & P25-P75 & Median & P25-P75 & & Median & P25-P75 & Median & P25-P75 & & \\
\hline Energy (MJ) & $6 \cdot 2$ & $5 \cdot 0-7 \cdot 6$ & $9 \cdot 1$ & $6 \cdot 9-12 \cdot 8$ & $<0.001$ & 9.0 & $6.5-11.6$ & 11.7 & $8.9-14.9$ & $<0.001$ & 0.049 \\
\hline Total protein $(\mathrm{g})$ & $40 \cdot 4$ & $31.9-51.0$ & $60 \cdot 4$ & $44 \cdot 6-82.5$ & $<0.001$ & $63 \cdot 2$ & $47 \cdot 4-87 \cdot 4$ & 86.5 & $64 \cdot 2-113.9$ & $<0.001$ & 0.808 \\
\hline Plant protein (g) & $27 \cdot 1$ & $20 \cdot 9-33 \cdot 2$ & $33 \cdot 6$ & $24 \cdot 3-48 \cdot 9$ & $<0.001$ & $31 \cdot 1$ & $22 \cdot 8-40 \cdot 4$ & 36.5 & $26 \cdot 7-49 \cdot 3$ & $<0.001$ & 0.039 \\
\hline Animal protein (g) & 12.5 & $7 \cdot 3-18.5$ & $24 \cdot 1$ & $14 \cdot 1-37 \cdot 1$ & $<0.001$ & 29.2 & $21 \cdot 4-40.9$ & $46 \cdot 7$ & $30 \cdot 9-65.9$ & $<0.001$ & 0.055 \\
\hline Total fat $(\mathrm{g})$ & $32 \cdot 1$ & $23 \cdot 2-42 \cdot 4$ & $56 \cdot 6$ & $36 \cdot 6-86 \cdot 7$ & $<0.001$ & 64.7 & $45 \cdot 9-88.4$ & 83.5 & $58 \cdot 3-112 \cdot 4$ & $<0.001$ & 0.003 \\
\hline Saturated fat $(\mathrm{g})$ & $7 \cdot 0$ & $4 \cdot 6-9 \cdot 8$ & $15 \cdot 4$ & $9 \cdot 6-24.5$ & $<0.001$ & $16 \cdot 6$ & $11 \cdot 3-23 \cdot 0$ & $22 \cdot 9$ & $15 \cdot 3-31 \cdot 4$ & $<0.001$ & $<0.001$ \\
\hline Monounsaturated fat (g) & $7 \cdot 1$ & $4.9-10.7$ & $15 \cdot 9$ & $9 \cdot 8-24 \cdot 6$ & $<0.001$ & $18 \cdot 1$ & $12 \cdot 2-26 \cdot 2$ & $25 \cdot 7$ & $16 \cdot 7-36 \cdot 5$ & $<0.001$ & 0.026 \\
\hline Polyunsaturated fat (g) & $10 \cdot 3$ & $7 \cdot 0-14.7$ & $17 \cdot 7$ & $10 \cdot 9-27 \cdot 7$ & $<0.001$ & $18 \cdot 3$ & $12 \cdot 6-25 \cdot 8$ & $22 \cdot 6$ & $16 \cdot 0-33 \cdot 7$ & $<0.001$ & 0.002 \\
\hline Cholesterol (mg) & 102 & 60-157 & 167 & 83313 & $<0.001$ & 234.9 & $152 \cdot 9-334.9$ & $342 \cdot 4$ & $215 \cdot 1-509 \cdot 8$ & $<0.001$ & 0.018 \\
\hline Total carbohydrate (g) & 243.5 & $191 \cdot 3-295 \cdot 6$ & $322 \cdot 0$ & $240 \cdot 9-468 \cdot 8$ & $<0.001$ & 294.6 & $209 \cdot 8-376 \cdot 2$ & 368.3 & $274.9-477.7$ & $<0.001$ & 0.103 \\
\hline Added sugar $(\mathrm{g})$ & 23.9 & $12 \cdot 8-36 \cdot 5$ & $46 \cdot 6$ & $24 \cdot 2-83 \cdot 6$ & $<0.001$ & $40 \cdot 6$ & $24 \cdot 1-62 \cdot 1$ & 67.6 & $32.6-98.5$ & $<0.001$ & 0.201 \\
\hline Dietary fibre (g) & $17 \cdot 3$ & $13 \cdot 8-22 \cdot 1$ & $20 \cdot 7$ & $14 \cdot 9-31 \cdot 3$ & $<0.001$ & $22 \cdot 8$ & $15 \cdot 1-30 \cdot 6$ & 27.5 & $19 \cdot 6-37 \cdot 8$ & $<0.001$ & 0.659 \\
\hline $\mathrm{Ca}(\mathrm{mg})$ & 198 & $129-277$ & 344 & $200-514$ & $<0.001$ & 402 & $282-622$ & 526 & $372-740$ & $<0.001$ & 0.050 \\
\hline $\mathrm{Fe}(\mathrm{mg})$ & $11 \cdot 2$ & $8.9-13.9$ & $15 \cdot 5$ & $11.2-21.6$ & $<0.001$ & 13.8 & $9.5-18.7$ & $18 \cdot 7$ & $13 \cdot 8-25 \cdot 2$ & $<0.001$ & 0.526 \\
\hline $\operatorname{Mg}(\mu \mathrm{g})$ & 240 & $188-310$ & 365 & $270-522$ & $<0.001$ & 331 & $237-424$ & 402 & $300-548$ & $<0.001$ & 0.004 \\
\hline$P(\mathrm{mg})$ & 694 & $544-876$ & 1054 & $788-1413$ & $<0.001$ & 1018 & $744-1361$ & 1292 & $976-1751$ & $<0.001$ & 0.063 \\
\hline $\mathrm{K}(\mathrm{mg})$ & 1223 & $991-1546$ & 2322 & $1742-3213$ & $<0.001$ & 2088 & $1394-2581$ & 2948 & 2208-3898 & $<0.001$ & 0.063 \\
\hline $\mathrm{Zn}(\mathrm{mg})$ & 7.9 & $6 \cdot 2-9 \cdot 6$ & $12 \cdot 4$ & $9 \cdot 0-17 \cdot 3$ & $<0.001$ & $10 \cdot 8$ & $7 \cdot 5-14.8$ & $15 \cdot 7$ & $11 \cdot 8-20 \cdot 9$ & $<0.001$ & 0.302 \\
\hline $\mathrm{Cu}(\mathrm{mg})$ & 1.0 & $0.8-1.3$ & 1.4 & $1.0-1.9$ & $<0.001$ & 1.4 & $1.0-1.9$ & 1.7 & $1 \cdot 3-2 \cdot 4$ & $<0.001$ & 0.275 \\
\hline $\mathrm{Mn}(\mathrm{mg})$ & 1221 & $783-1876$ & 1820 & $1296-2524$ & $<0.001$ & 2252 & $1532-3152$ & 2650 & $1804-3618$ & $<0.001$ & 0.112 \\
\hline Vitamin A $(\mu \mathrm{g})$ & 476 & $336-704$ & 959 & 609-1575 & $<0.001$ & 995 & $560-1563$ & 1724 & $985-2588$ & $<0.001$ & 0.241 \\
\hline Thiamin (mg) & 1.4 & $1 \cdot 1-1 \cdot 8$ & 1.8 & $1 \cdot 3-2 \cdot 6$ & $<0.001$ & 1.5 & $1 \cdot 1-2 \cdot 2$ & 2.0 & $1.6-2.9$ & $<0.001$ & 0.843 \\
\hline Riboflavin (mg) & 0.7 & $0.6-1.0$ & 1.2 & $0.9-1.7$ & $<0.001$ & 1.4 & $0.9-2.0$ & 1.9 & $1 \cdot 4-2 \cdot 7$ & $<0.001$ & 0.605 \\
\hline Niacin (mg) & $10 \cdot 4$ & $8 \cdot 4-13 \cdot 6$ & $22 \cdot 2$ & $15 \cdot 7-30 \cdot 6$ & $<0.001$ & $16 \cdot 1$ & $11 \cdot 3-22 \cdot 3$ & $28 \cdot 2$ & $21.0-38.9$ & $<0.001$ & 0.788 \\
\hline Vitamin $B_{6}(\mathrm{mg})$ & 1.2 & $0.9-1.5$ & $2 \cdot 2$ & $1.5-3.8$ & $<0.001$ & 1.6 & $1 \cdot 1-2 \cdot 3$ & 3.4 & $2.5-4.7$ & $<0.001$ & $<0.001$ \\
\hline Folate $(\mu \mathrm{g})$ & 335 & $253-429$ & 424 & $260-663$ & $<0.001$ & 374 & $272-531$ & 533 & $375-781$ & $<0.001$ & 0.054 \\
\hline Vitamin $B_{12}(\mu \mathrm{g})$ & 1.7 & $0.8-3.0$ & 2.4 & $1 \cdot 2-5 \cdot 2$ & $<0.001$ & 4.6 & $2.7-7.5$ & $5 \cdot 6$ & $3 \cdot 1-10 \cdot 6$ & $<0.001$ & 0.876 \\
\hline Pantothenic acid (mg) & $2 \cdot 6$ & $2.0-3.5$ & 4.3 & $3 \cdot 1-6 \cdot 6$ & $<0.001$ & 4.7 & $3 \cdot 4-6 \cdot 2$ & $6 \cdot 8$ & $5 \cdot 4-9.5$ & $<0.001$ & 0.003 \\
\hline Biotin $(\mu \mathrm{g})$ & $22 \cdot 2$ & $16 \cdot 9-31 \cdot 7$ & $29 \cdot 2$ & $20 \cdot 1-44 \cdot 5$ & $<0.001$ & $42 \cdot 8$ & $29 \cdot 8-58 \cdot 3$ & 58.7 & $38 \cdot 6-78 \cdot 7$ & $<0.001$ & 0.002 \\
\hline Vitamin C (mg) & 13 & 8-19 & 32 & $18-55$ & $<0.001$ & 40 & $21-59$ & 64 & $40-107$ & $<0.001$ & 0.132 \\
\hline Vitamin D (mg) & 1.6 & $0 \cdot 8-2 \cdot 6$ & 1.9 & $0.9-0.9$ & $<0.001$ & $2 \cdot 8$ & $1.6-4.7$ & 4.2 & $2 \cdot 5-6 \cdot 6$ & $<0.001$ & 0.045 \\
\hline Vitamin E (mg) & 7.9 & $5 \cdot 6-12 \cdot 1$ & 11.2 & $6 \cdot 9-16 \cdot 7$ & $<0.001$ & $12 \cdot 4$ & $8 \cdot 4-16 \cdot 9$ & 14.7 & $9 \cdot 6-21 \cdot 7$ & $<0.001$ & 0.307 \\
\hline Alcohol (g) & & $0.0-0.0$ & 0.0 & $0.0-0.0$ & 0.017 & 0.0 & $0.0-14.3$ & 0.0 & $0 \cdot 0-6 \cdot 1$ & 0.092 & 0.825 \\
\hline \multicolumn{12}{|l|}{ Energy distribution } \\
\hline$\%$ of TE from protein & $10 \cdot 9$ & $9 \cdot 9-12 \cdot 0$ & $11 \cdot 1$ & $9 \cdot 6-12 \cdot 9$ & 0.326 & 12.5 & $11 \cdot 3-13 \cdot 5$ & 12.5 & $11 \cdot 1-14 \cdot 3$ & 0.025 & 0.315 \\
\hline$\%$ of TE from animal protein & 3.3 & $2 \cdot 2-4 \cdot 8$ & 4.5 & $2 \cdot 8-6 \cdot 7$ & $<0.001$ & $5 \cdot 8$ & $4 \cdot 7-7 \cdot 2$ & $6 \cdot 8$ & $5 \cdot 1-9 \cdot 2$ & $<0.001$ & 0.817 \\
\hline$\%$ of TE from plant protein & 7.5 & $6.6-8.0$ & $6 \cdot 3$ & $5 \cdot 4-7 \cdot 4$ & $<0.001$ & $6 \cdot 1$ & $5 \cdot 3-6 \cdot 8$ & 5.5 & $4 \cdot 6-6 \cdot 2$ & $<0.001$ & $<0.001$ \\
\hline$\%$ of TE from total fat & $20 \cdot 3$ & $15 \cdot 6-24.5$ & $22 \cdot 9$ & $17 \cdot 7-30 \cdot 0$ & $<0.001$ & 28.4 & $23.7-31.9$ & $27 \cdot 7$ & $22 \cdot 8-32 \cdot 3$ & 0.335 & $<0.001$ \\
\hline$\%$ of TE from saturated fat & 4.3 & $2 \cdot 9-5 \cdot 7$ & 6.5 & $4.6-8.5$ & $<0.001$ & 7.0 & $5 \cdot 8-8 \cdot 6$ & 7.5 & $5 \cdot 9-9 \cdot 0$ & 0.130 & $<0.001$ \\
\hline$\%$ of TE from monounsaturated fat & 4.5 & $3 \cdot 2-5 \cdot 9$ & $6 \cdot 2$ & $4.6-8.7$ & $<0.001$ & 7.9 & $6 \cdot 4-9 \cdot 8$ & 8.4 & $6 \cdot 6-10 \cdot 5$ & 0.019 & $<0.001$ \\
\hline$\%$ of TE from polyunsaturated fat & 6.5 & $4.8-8.6$ & $7 \cdot 3$ & 4.9-9.9 & $<0.001$ & 8.0 & $6 \cdot 4-9 \cdot 7$ & $7 \cdot 6$ & $6 \cdot 1-9 \cdot 3$ & 0.125 & $<0.001$ \\
\hline$\%$ of TE from total carbohydrate & $66 \cdot 7$ & $61.3-71.9$ & $61 \cdot 7$ & $53 \cdot 8-67 \cdot 7$ & $<0.001$ & $55 \cdot 7$ & $51 \cdot 1-60 \cdot 2$ & $54 \cdot 1$ & $49 \cdot 5-59 \cdot 8$ & 0.039 & $<0.001$ \\
\hline$\%$ of TE from added sugar & $6 \cdot 4$ & $3 \cdot 7-10 \cdot 2$ & 8.9 & $4.3-14.9$ & $<0.001$ & 8.2 & $5 \cdot 7-10 \cdot 8$ & $9 \cdot 0$ & $5 \cdot 9-14.5$ & $<0.001$ & 0.055 \\
\hline$\%$ of TE from alcohol & 0.0 & $0.0-0.0$ & 0.0 & $0.0-0.0$ & $<0.001$ & 0.0 & $0.0-4.4$ & 0.0 & $0.0-1.5$ & 0.002 & 0.731 \\
\hline
\end{tabular}


When expressed as a percentage of energy, the differences in change of macronutrients were significant for plant protein, total, saturated, monounsaturated and polyunsaturated fats, and total carbohydrate, with the rural women showing the largest change. Significant differences in change of micronutrients expressed per energy are also shown in Table 3. For dietary fibre, these changes showed a decrease in rural women but an increase in urban women. Therefore, the direction of change in rural and urban women was not always the same when nutrients were expressed in terms of energy intake.

\section{Evaluation of dietary quality}

We also measured how well the cohort participants met their nutrient needs and dietary recommendations for the prevention of NCD. The recommendations in Supplemental Table 1 (see online supplementary material) were used to determine whether a particular nutrient was under- or overconsumed by each participant. Furthermore, the percentage of participants in specific groups who did not meet their recommended intakes was also calculated. Table 4 gives values for men and Table 5 those for women, both by date (2005 and 2010) and rural $v$. urban participants.

Table 4 shows that in 2005 more than $50 \%$ of the rural men did not meet the recommendation for sixteen of the twenty-three nutrients presented. More than $50 \%$ of the urban men did not meet the recommendations for only six of the twenty-three nutrients in 2005. Although the situation improved in 2010 for the rural men, more than $50 \%$ of them did not meet the recommendations for cholesterol, dietary fibre, Ca, K, pantothenic acid, vitamin C, vitamin D and vitamin E. Despite a higher energy intake (meaning that more food was eaten), still more than $50 \%$ of the urban men had low $\mathrm{Ca}, \mathrm{K}$, vitamin $\mathrm{C}$ and vitamin $\mathrm{D}$ intakes in 2010 which did not meet the recommendations. In addition, the energy distribution was not optimal. There was a significant improvement in intake compared with the recommendation for rural and urban men for total protein, cholesterol, $\mathrm{Zn}, \mathrm{Cu}, \mathrm{Mn}$, vitamin $\mathrm{A}$, riboflavin, niacin, vitamin $\mathrm{B}_{6}$, pantothenic acid, biotin, vitamin $\mathrm{C}$, vitamin $\mathrm{D}$ and vitamin $\mathrm{E}$.

Table 5 shows that in $2005,50 \%$ of the rural women did not meet the recommendation for seventeen of the twenty-three nutrients presented. Although there was a significant improvement from 2005 to 2010, more than $50 \%$ of the rural women still had an intake not meeting the recommendation for nine nutrients (cholesterol, fibre, Ca, $\mathrm{K}$, pantothenic acid, biotin, vitamin $\mathrm{C}$, vitamin $\mathrm{D}$ and vitamin E) in 2010. The nutrient quality of the diet of the urban women was better than that of the rural women, with more than $50 \%$ of the urban women not meeting the recommendation for only seven of the twenty-three nutrients in 2005 and for only three nutrients (Ca, K and vitamin D) in 2010. 
Table 4 Nutrient intakes of the male cohort, expressed as a percentage of the recommendation, and the percentage who did not meet recommendations by urban/rural residence in 2005 and 2010; PURE-NWP-SA study

\begin{tabular}{|c|c|c|c|c|c|c|c|c|c|c|c|c|c|c|}
\hline \multirow[b]{3}{*}{ Nutrients } & \multicolumn{6}{|c|}{ Rural men ( $n$ 186) } & \multirow[b]{3}{*}{$P$ value } & \multicolumn{6}{|c|}{ Urban men (n 202) } & \multirow[b]{3}{*}{$P$ value } \\
\hline & \multicolumn{3}{|c|}{2005} & \multicolumn{3}{|c|}{2010} & & \multicolumn{3}{|c|}{2005} & \multicolumn{3}{|c|}{2010} & \\
\hline & Median & P25-P75 & $\%^{*}$ & Median & P25-P75 & $\%^{*}$ & & Median & P25-P75 & $\%^{*}$ & Median & P25-P75 & $\%^{*}$ & \\
\hline Total protein & $79 \cdot 3$ & $63 \cdot 1-109 \cdot 2$ & 69.9 & $118 \cdot 2$ & $83 \cdot 6-163 \cdot 3$ & 37.6 & $<0.001$ & 129.9 & $93 \cdot 8-171 \cdot 0$ & 30.7 & 178.5 & $134 \cdot 0-247 \cdot 2$ & $10 \cdot 4$ & $<0.001$ \\
\hline Cholesterol & 37 & $24-57$ & $95 \cdot 2$ & $70 \cdot 1$ & $29 \cdot 1-125 \cdot 7$ & 64.5 & $<0.001$ & 83.1 & $54.4-118.3$ & $66 \cdot 8$ & 136.0 & $92.9-219.1$ & $28 \cdot 7$ & $<0.001$ \\
\hline Dietary fibre & 73.9 & $56 \cdot 8-100 \cdot 7$ & 74.7 & $82 \cdot 4$ & $56 \cdot 7-124 \cdot 6$ & 64.0 & 0.020 & $109 \cdot 0$ & $70 \cdot 1-141 \cdot 6$ & $47 \cdot 0$ & $125 \cdot 7$ & $92 \cdot 4-172 \cdot 5$ & $31 \cdot 2$ & $<0.001$ \\
\hline $\mathrm{Ca}$ & 28.4 & $19 \cdot 3-42 \cdot 8$ & 98.9 & $42 \cdot 0$ & $24 \cdot 0-66 \cdot 2$ & 94.1 & 0.013 & 49.2 & $36 \cdot 0-71.5$ & 89.6 & 71.8 & $53.3-99.7$ & $75 \cdot 3$ & $<0.001$ \\
\hline $\mathrm{Fe}$ & 203.6 & $151 \cdot 1-265 \cdot 0$ & 6.5 & 265.9 & $189 \cdot 5-385 \cdot 7$ & 4.3 & 0.346 & $266 \cdot 3$ & $186 \cdot 8-364 \cdot 2$ & 6.4 & 369.1 & $270 \cdot 3-500 \cdot 9$ & 0.0 & $<0.001$ \\
\hline $\mathrm{Mg}$ & 84.8 & $61 \cdot 3-129 \cdot 1$ & 61.3 & 114.8 & $81 \cdot 4-166.0$ & 38.7 & $<0.001$ & 111.1 & $82 \cdot 2-153 \cdot 9$ & $38 \cdot 1$ & $140 \cdot 8$ & $105 \cdot 4-212 \cdot 9$ & $20 \cdot 3$ & $<0.001$ \\
\hline $\mathrm{P}^{\circ}$ & 146.0 & $111.4-191.8$ & $18 \cdot 3$ & 208.0 & $144 \cdot 6-282 \cdot 4$ & $9 \cdot 1$ & 0.007 & 190.9 & $147 \cdot 6-254.7$ & 9.4 & 283.1 & $201 \cdot 0-384 \cdot 4$ & 1.5 & $<0.001$ \\
\hline $\mathrm{K}$ & 29.7 & $23 \cdot 2-37 \cdot 4$ & $100 \cdot 0$ & 51.9 & $36 \cdot 4-69 \cdot 0$ & $94 \cdot 1$ & $<0.001$ & $44 \cdot 2$ & $32.5-59.8$ & 97.5 & 70.8 & $51.5-93.1$ & $80 \cdot 7$ & $<0.001$ \\
\hline $\mathrm{Zn}$ & 91.9 & $70.7-119.8$ & 55.4 & 141.6 & $100.5-214.9$ & 24.7 & $<0.001$ & $133 \cdot 3$ & $95 \cdot 3-181 \cdot 6$ & $27 \cdot 2$ & $196 \cdot 0$ & $150 \cdot 2-281 \cdot 9$ & 5.9 & $<0.001$ \\
\hline $\mathrm{Cu}$ & 153.4 & $114.3-215 \cdot 9$ & $14 \cdot 0$ & 198.1 & $133 \cdot 8-278.6$ & $9 \cdot 1$ & 0.150 & 211.4 & $151 \cdot 5-269 \cdot 7$ & $10 \cdot 9$ & 272.6 & $202 \cdot 9-375 \cdot 3$ & 0.5 & $<0.001$ \\
\hline $\mathrm{Mn}$ & 76.1 & $48 \cdot 2-141 \cdot 7$ & 63.4 & 89.6 & $57 \cdot 8-135.8$ & 53.2 & 0.042 & 115.1 & $82 \cdot 6-168 \cdot 1$ & $42 \cdot 6$ & $136 \cdot 2$ & $96.5-182.1$ & $27 \cdot 2$ & $<0.001$ \\
\hline Vitamin A & 68.3 & $43.9-113.8$ & $70 \cdot 4$ & $150 \cdot 5$ & $85 \cdot 6-276 \cdot 3$ & 30.7 & $<0.001$ & $142 \cdot 4$ & $85.7-231.5$ & $33 \cdot 2$ & 278.7 & $166 \cdot 8-411 \cdot 6$ & 5.5 & $<0.001$ \\
\hline Thiamin & 158.7 & $119 \cdot 4-206 \cdot 2$ & 14.5 & $190 \cdot 3$ & $128 \cdot 1-283 \cdot 7$ & $10 \cdot 8$ & 0.250 & 190.9 & $134 \cdot 1-273 \cdot 4$ & $12 \cdot 9$ & 245.4 & $183 \cdot 6-370 \cdot 1$ & 3.5 & $<0.001$ \\
\hline Riboflavin & 84.4 & $61.9-128.8$ & 63.4 & 131.0 & $88.4-179.1$ & 32.8 & $<0.001$ & $136 \cdot 3$ & $95 \cdot 6-186 \cdot 4$ & $26 \cdot 2$ & $206 \cdot 6$ & $140 \cdot 3-281 \cdot 5$ & 7.9 & $<0.001$ \\
\hline Niacin & 101.4 & $78.0-146.5$ & 48.9 & $200 \cdot 7$ & $142 \cdot 0-290 \cdot 1$ & $7 \cdot 0$ & $<0.001$ & $154 \cdot 3$ & $116 \cdot 8-209 \cdot 4$ & $19 \cdot 3$ & 286.6 & $209 \cdot 7-414.0$ & 0.0 & $<0.001$ \\
\hline Vitamin $B_{6}$ & 101.5 & $78 \cdot 1-128 \cdot 8$ & $46 \cdot 8$ & 202.0 & $124 \cdot 9-305 \cdot 2$ & 8.6 & $<0.001$ & $157 \cdot 1$ & $95 \cdot 1-210 \cdot 7$ & 28.2 & 331.8 & $209.2-475.9$ & 1.0 & $<0.001$ \\
\hline Folate & 111.6 & $84 \cdot 0-146 \cdot 1$ & 39.8 & $146 \cdot 1$ & $94.0-232 \cdot 8$ & 29.0 & 0.027 & 138.4 & $96 \cdot 0-195 \cdot 8$ & $26 \cdot 2$ & 211.2 & $151.7-319.6$ & 8.4 & $<0.001$ \\
\hline Vitamin $B_{12}$ & 88.0 & $45 \cdot 3-147 \cdot 6$ & $59 \cdot 1$ & 129.6 & $65 \cdot 8-275 \cdot 9$ & 39.8 & $<0.001$ & 238.8 & $118 \cdot 2-385 \cdot 1$ & $17 \cdot 3$ & $306 \cdot 0$ & $180 \cdot 2-564 \cdot 2$ & 9.4 & 0.014 \\
\hline Pantothenic acid & 57.4 & $44.8-81.5$ & $86 \cdot 0$ & 92.0 & $62 \cdot 9-130 \cdot 2$ & 55.4 & $<0.001$ & 98.5 & $71 \cdot 3-131 \cdot 1$ & $51 \cdot 0$ & $172 \cdot 1$ & $124 \cdot 0-228.6$ & $15 \cdot 8$ & $<0.001$ \\
\hline Biotin & 83.4 & $60 \cdot 8-106 \cdot 4$ & $67 \cdot 7$ & $102 \cdot 7$ & $64 \cdot 1-161 \cdot 4$ & 48.4 & $<0.001$ & 152.6 & $98 \cdot 3-205 \cdot 7$ & $25 \cdot 3$ & $208 \cdot 3$ & $139.5-295.8$ & 9.9 & $<0.001$ \\
\hline Vitamin C & $15 \cdot 3$ & $9 \cdot 0-22 \cdot 0$ & 99.5 & 39.1 & $18 \cdot 1-74 \cdot 8$ & 85.0 & $<0.001$ & 43.8 & $26 \cdot 5-76 \cdot 5$ & 84.2 & $82 \cdot 7$ & $48.0-128.8$ & 58.4 & $<0.001$ \\
\hline Vitamin D & $17 \cdot 1$ & $8.9-26.5$ & 98.9 & 23.8 & $9 \cdot 6-51 \cdot 2$ & $90 \cdot 3$ & $<0.001$ & 32.0 & $19 \cdot 8-48 \cdot 1$ & 97.5 & $50 \cdot 2$ & $27 \cdot 5-81 \cdot 7$ & $82 \cdot 2$ & $<0.001$ \\
\hline Vitamin E & 69.8 & $43.0-99.3$ & 75.8 & 98.0 & $48 \cdot 8-162 \cdot 7$ & 51.6 & $<0.001$ & 103.4 & $68 \cdot 9-145 \cdot 4$ & 48.5 & 128.5 & $87 \cdot 8-196 \cdot 9$ & 30.2 & $<0.001$ \\
\hline \multicolumn{15}{|l|}{ Energy distribution } \\
\hline$\%$ of TE from fat & $59 \cdot 3$ & $45 \cdot 2-76.9$ & $94 \cdot 6$ & 71.6 & $52 \cdot 2-96 \cdot 6$ & $78 \cdot 0$ & $<0.001$ & 83.1 & $72 \cdot 1-98 \cdot 1$ & $77 \cdot 2$ & 87.8 & $73 \cdot 6-104 \cdot 4$ & $69 \cdot 3$ & 0.056 \\
\hline$\%$ of TE from saturated fat & 38.5 & $25 \cdot 1-50 \cdot 6$ & $96 \cdot 2$ & 62.1 & $41.3-84.5$ & $90 \cdot 3$ & 0.016 & 64.5 & $45 \cdot 0-91 \cdot 8$ & 93.6 & 68.8 & $56 \cdot 5-87 \cdot 2$ & $87 \cdot 6$ & 0.034 \\
\hline$\%$ of TE from polyunsaturated fat & 99.7 & $69.4-131.9$ & 51.6 & 101.6 & $70 \cdot 6-148 \cdot 8$ & 49.5 & 0.689 & $119 \cdot 4$ & $95 \cdot 5-148 \cdot 2$ & $29 \cdot 2$ & $120 \cdot 1$ & $94 \cdot 6-151 \cdot 7$ & 29.2 & 1.000 \\
\hline$\%$ of TE from added sugar & $58 \cdot 3$ & $29.9-82.9$ & $85 \cdot 0$ & $80 \cdot 7$ & $45 \cdot 4-142 \cdot 4$ & $57 \cdot 0$ & $<0.001$ & 63.6 & $51 \cdot 1-75 \cdot 5$ & $80 \cdot 2$ & $79 \cdot 7$ & $45 \cdot 4-120 \cdot 3$ & $64 \cdot 4$ & $<0.001$ \\
\hline
\end{tabular}

PURE-NWP-SA, Prospective Urban and Rural Epidemiology in the North West Province of South Africa; P25, 25th percentile; P75, 75th percentile; TE, total energy.

*Percentage of participants not meeting the recommendations.
$\dagger P$ value for significance of differences between 2005 and 2010 for rural men or urban men not meeting the recommendations (Wilcoxon two-sample test) 
Table 5 Nutrient intakes of the female cohort, expressed as a percentage of the recommendation, and percentage who did not meet recommendations by urban/rural residence in 2005 and 2010; PURE-NWP-SA study

\begin{tabular}{|c|c|c|c|c|c|c|c|c|c|c|c|c|c|c|}
\hline \multirow[b]{3}{*}{ Nutrients } & \multicolumn{6}{|c|}{ Rural women ( $n 411)$} & \multirow[b]{3}{*}{$P$ valuet } & \multicolumn{6}{|c|}{ Urban women (n 355) } & \multirow[b]{3}{*}{$P$ value $\dagger$} \\
\hline & \multicolumn{3}{|c|}{2005} & \multicolumn{3}{|c|}{2010} & & \multicolumn{3}{|c|}{2005} & \multicolumn{3}{|c|}{2010} & \\
\hline & Median & P25-P75 & $\%^{*}$ & Median & P25-P75 & $\% *$ & & Median & P25-P75 & $\%^{*}$ & Median & P25-P75 & $\% *$ & \\
\hline Total protein & 87.9 & $69 \cdot 4-110 \cdot 9$ & $63 \cdot 3$ & 131.4 & $97 \cdot 0-179 \cdot 3$ & $27 \cdot 3$ & $<0.001$ & 137.6 & $103 \cdot 1-190 \cdot 0$ & $22 \cdot 3$ & $188 \cdot 1$ & $139 \cdot 6-247 \cdot 7$ & 9.6 & $<0.001$ \\
\hline Cholesterol & 33.9 & $20 \cdot 1-52 \cdot 3$ & 93.2 & 55.8 & $27 \cdot 8-104 \cdot 5$ & 73.7 & $<0.001$ & 78.3 & $51.0-111.6$ & $66 \cdot 8$ & 114.1 & $71.7-169.9$ & 39.4 & $<0.001$ \\
\hline Dietary fibre & $69 \cdot 3$ & $55 \cdot 0-88.4$ & $87 \cdot 6$ & 83.0 & $59 \cdot 9-125.0$ & $62 \cdot 3$ & $<0.001$ & 91.0 & $60 \cdot 4-122.5$ & $56 \cdot 9$ & 109.9 & $78 \cdot 3-151 \cdot 1$ & $42 \cdot 3$ & $<0.001$ \\
\hline $\mathrm{Ca}$ & 22.9 & $14.7-31.6$ & 98.5 & 38.0 & $22.5-57.5$ & 94.4 & 0.002 & 46.0 & $30 \cdot 9-70 \cdot 7$ & 93.0 & 56.2 & $39 \cdot 1-82 \cdot 4$ & 85.1 & $<0.001$ \\
\hline $\mathrm{Fe}$ & $166 \cdot 0$ & $123 \cdot 8-216 \cdot 8$ & 10.5 & $250 \cdot 3$ & $176 \cdot 8-352 \cdot 0$ & 3.4 & $<0.001$ & 206.0 & $144.7-305.9$ & $9 \cdot 6$ & $316 \cdot 1$ & $226 \cdot 8-431 \cdot 4$ & 1.1 & $<0.001$ \\
\hline $\mathrm{Mg}$ & 90.5 & $71 \cdot 0-116.9$ & 59.9 & $137 \cdot 8$ & $101 \cdot 8-196 \cdot 8$ & 23.1 & $<0.001$ & 125.0 & $89.4-159.9$ & 31.0 & 151.8 & $113 \cdot 1-206 \cdot 7$ & $15 \cdot 5$ & $<0.001$ \\
\hline P & 119.7 & $93.9-151.0$ & 29.7 & 181.7 & $135 \cdot 9-243 \cdot 6$ & $6 \cdot 1$ & $<0.001$ & 175.5 & $128 \cdot 2-234 \cdot 7$ & 10.7 & 222.7 & $168 \cdot 3-301 \cdot 8$ & 3.1 & $<0.001$ \\
\hline $\mathrm{K}$ & $26 \cdot 0$ & $21 \cdot 1-32 \cdot 9$ & 99.8 & 49.4 & $37 \cdot 1-68 \cdot 4$ & 93.4 & $<0.001$ & 44.4 & $29.7-54.9$ & 98.9 & 62.7 & $47 \cdot 0-82 \cdot 9$ & 85.6 & $<0.001$ \\
\hline $\mathrm{Zn}$ & $115 \cdot 8$ & $91.5-141.8$ & 33.6 & 182.0 & $132 \cdot 8-254 \cdot 3$ & $7 \cdot 3$ & $<0.001$ & 159.5 & $110.9-217.4$ & $18 \cdot 0$ & $231 \cdot 3$ & $173 \cdot 4-308 \cdot 1$ & 5.4 & $<0.001$ \\
\hline $\mathrm{Cu}$ & 141.6 & $112 \cdot 0-178.6$ & 73.7 & 198.5 & $140 \cdot 6-269 \cdot 4$ & 7.5 & $<0.001$ & $196 \cdot 2$ & $143 \cdot 3-272 \cdot 8$ & 34.4 & 243.3 & $178 \cdot 2-345 \cdot 3$ & 3.9 & 0.002 \\
\hline $\mathrm{Mn}$ & 67.8 & $43.5-104.2$ & $16 \cdot 6$ & $101 \cdot 1$ & $72 \cdot 0-140 \cdot 2$ & $49 \cdot 2$ & $<0.001$ & $125 \cdot 1$ & $85 \cdot 1-175 \cdot 1$ & $9 \cdot 6$ & $147 \cdot 2$ & $100 \cdot 2-201 \cdot 0$ & 24.8 & 0.004 \\
\hline Vitamin A & $95 \cdot 2$ & $67 \cdot 2-140 \cdot 8$ & $53 \cdot 3$ & $192 \cdot 0$ & $121 \cdot 8-315 \cdot 1$ & 18.0 & $<0.001$ & 198.9 & $112 \cdot 1-312 \cdot 6$ & 21.4 & 344.8 & $197 \cdot 1-517 \cdot 6$ & 3.1 & $<0.001$ \\
\hline Thiamin & 158.7 & $126 \cdot 0-200 \cdot 9$ & 8.0 & $200 \cdot 0$ & $141.9-287.0$ & 9.3 & 0.529 & 170.0 & $124 \cdot 4-248.7$ & $12 \cdot 7$ & 226.5 & $172 \cdot 0-317 \cdot 2$ & 4.2 & $<0.001$ \\
\hline Riboflavin & 80.9 & $62.1-112.5$ & 68.1 & 135.9 & $99.4-193.4$ & $25 \cdot 3$ & $<0.001$ & $156 \cdot 4$ & $108 \cdot 9-223 \cdot 1$ & $21 \cdot 7$ & $221 \cdot 1$ & $155 \cdot 2-298 \cdot 4$ & 7.6 & $<0.001$ \\
\hline Niacin & 94.6 & $76 \cdot 5-123 \cdot 4$ & $55 \cdot 2$ & 201.3 & $142 \cdot 9-278.6$ & $6 \cdot 6$ & $<0.001$ & $146 \cdot 1$ & $102 \cdot 5-202 \cdot 7$ & $22 \cdot 8$ & 256.0 & $191 \cdot 3-353 \cdot 7$ & 2.0 & $<0.001$ \\
\hline Vitamin $B_{6}$ & 98.6 & $77 \cdot 8-129 \cdot 0$ & $50 \cdot 6$ & 184.8 & $125 \cdot 2-297.8$ & 11.4 & $<0.001$ & 130.0 & $86 \cdot 5-190 \cdot 3$ & $30 \cdot 7$ & 289.8 & $198.0-394.6$ & 2.0 & $<0.001$ \\
\hline Folate & 104.6 & $79 \cdot 1-134 \cdot 0$ & $45 \cdot 7$ & $132 \cdot 6$ & $81 \cdot 1-207 \cdot 1$ & 34.8 & $<0.001$ & 116.9 & $84.8-165.9$ & $36 \cdot 6$ & $166 \cdot 5$ & $117 \cdot 1-244.0$ & $16 \cdot 1$ & $<0.001$ \\
\hline Vitamin $B_{12}$ & 82.9 & $40 \cdot 5-150 \cdot 7$ & $56 \cdot 9$ & $120 \cdot 1$ & $62 \cdot 2-258 \cdot 7$ & 41.6 & $<0.001$ & 229.8 & $133.7-374.6$ & $17 \cdot 8$ & 280.7 & $152 \cdot 3-532 \cdot 2$ & $12 \cdot 4$ & 0.042 \\
\hline Pantothenic acid & $52 \cdot 1$ & $40 \cdot 0-70 \cdot 5$ & 92.0 & $85 \cdot 8$ & $61 \cdot 4-131.0$ & $59 \cdot 6$ & $<0.001$ & 94.2 & $67 \cdot 6-123 \cdot 1$ & $56 \cdot 1$ & 136.8 & $108 \cdot 3-190 \cdot 9$ & 20.0 & $<0.001$ \\
\hline Biotin & $74 \cdot 1$ & $56 \cdot 3-105 \cdot 5$ & $72 \cdot 0$ & $97 \cdot 3$ & $66 \cdot 9-148 \cdot 2$ & 51.6 & $<0.001$ & $142 \cdot 6$ & $99.5-194.3$ & $25 \cdot 1$ & $195 \cdot 7$ & $128 \cdot 7-262.4$ & 14.9 & $<0.001$ \\
\hline Vitamin C & $20 \cdot 9$ & $13 \cdot 7-31 \cdot 5$ & $98 \cdot 1$ & $52 \cdot 6$ & $29 \cdot 4-90 \cdot 9$ & 78.4 & $<0.001$ & 66.3 & $35 \cdot 0-98 \cdot 6$ & $75 \cdot 2$ & $106 \cdot 8$ & $67.1-178.5$ & 46.8 & $<0.001$ \\
\hline Vitamin D & $15 \cdot 9$ & $8 \cdot 4-25 \cdot 6$ & $98 \cdot 8$ & 19.5 & $9 \cdot 3-45 \cdot 7$ & 94.4 & $<0.001$ & $27 \cdot 6$ & $15 \cdot 8-47 \cdot 3$ & $98 \cdot 3$ & 41.5 & $24 \cdot 8-66 \cdot 2$ & 91.8 & $<0.001$ \\
\hline Vitamin E & $66 \cdot 4$ & $46 \cdot 8-100 \cdot 6$ & 74.5 & $93 \cdot 1$ & $58 \cdot 0-139 \cdot 1$ & 53.3 & $<0.001$ & 103.6 & $70 \cdot 4-141.0$ & $46 \cdot 2$ & $122 \cdot 2$ & $80 \cdot 0-181 \cdot 1$ & 37.2 & 0.011 \\
\hline \multicolumn{15}{|l|}{ Energy distribution } \\
\hline$\%$ of TE from fat & 67.5 & $52 \cdot 0-81.7$ & $92 \cdot 0$ & $76 \cdot 2$ & $58 \cdot 9-100 \cdot 0$ & 74.9 & $<0.001$ & 94.6 & $78 \cdot 9-106 \cdot 5$ & $62 \cdot 8$ & $92 \cdot 2$ & $76 \cdot 1-107 \cdot 6$ & 61.1 & 0.629 \\
\hline$\%$ of TE from saturated fat & $64 \cdot 2$ & $36 \cdot 5-102 \cdot 0$ & 97.8 & 64.6 & $45 \cdot 6-85 \cdot 2$ & 88.1 & $<0.001$ & 81.8 & $56 \cdot 9-108 \cdot 2$ & 88.2 & $75 \cdot 0$ & $58 \cdot 8-90 \cdot 1$ & 83.4 & 0.065 \\
\hline$\%$ of TE from polyunsaturated fat & $107 \cdot 8$ & $80 \cdot 4-143 \cdot 2$ & 43.1 & $121 \cdot 7$ & $83 \cdot 2-166 \cdot 5$ & 33.8 & 0.004 & 133.5 & $107 \cdot 4-161 \cdot \overline{7}$ & $18 \cdot \overline{6}$ & $126 \cdot 9$ & $101 \cdot 9-154 \cdot 2$ & 22.5 & 0.186 \\
\hline$\%$ of TE from added sugar & $42 \cdot 8$ & $29 \cdot 9-56 \cdot 8$ & 74.2 & 89.8 & $42 \cdot 7-149 \cdot 0$ & $55 \cdot 7$ & $<0.001$ & $70 \cdot 2$ & $57 \cdot 5-85.7$ & 67.9 & $90 \cdot 3$ & $59 \cdot 4-144.7$ & 55.8 & $<0.001$ \\
\hline
\end{tabular}

PURE-NWP-SA, Prospective Urban and Rural Epidemiology in the North West Province of South Africa; P25, 25th percentile; P75, 75th percentile; TE, total energy.

*Percentage of participants not meeting the recommendations.

$\dagger P$ value for significance of differences between 2005 and 2010 for rural women or urban women not meeting the recommendations (Wilcoxon two-sample test) 
The energy distribution of the rural and urban women was not optimal. Because of overconsumption, more than $50 \%$ of the participants in 2005 and 2010 did not meet the restrictive recommendations for total fat, saturated fat and added sugar.

\section{Discussion}

The objectives of the present part of the PURE-NWP-SA study were to measure and report the changes in nutrient intakes of rural and urban black African men and women over time, to assess the impact of urbanization and modernization of lifestyles on dietary and therefore nutrient intakes and NCD risk.

\section{Energy intakes}

The first important result was the substantial increase in energy intake from 2005 to 2010 in all groups. Increases in median values were $2 \cdot 3$, 3.4, $2 \cdot 6$ and $2 \cdot 3 \mathrm{MJ} / \mathrm{d}$ for rural and urban men and rural and urban women, respectively. In 2010 (within 5 years) the rural subjects 'caught up' with the energy intake of the urban subjects measured in 2005. In 2010 the urban men consumed 13.3 MJ daily and women $11 \cdot 3 \mathrm{MJ}$ daily.

The question arises whether these increases are real or a result of methodological error. The facts argue against the latter. The same team of fieldworkers and researchers used in 2010 the same validated QFFQ that was used in 2005 to measure intakes. The reproducibility of the QFFQ was satisfactorily validated in the PURE-NWP-SA study in $2005^{(18)}$. Because the median energy values of rural men and women in 2010 were similar to those of urban men and women in 2005, this suggests that 'environmental' or lifestyle factors were responsible for the increased intakes in 2010. There are several known contenders: (i) after the 2005 measurements, a large grocery and food store opened in the rural area which increased the availability and affordability of many foods, processed food products and beverages; (ii) social support in the form of grants in both rural and urban areas, and thus income, also increased over this time; and (iii) the rural area experienced a severe drought in 2005 which limited the availability of fresh products and income to buy them.

It seems reasonable to conclude that the observed rapid increases in energy intake in both rural and urban participants were real and part of an accelerated nutrition transition. The increases in energy intake were of course accompanied by expected increases in intakes of all other nutrients because of greater food consumption.

Similarly, a consistent rise in total energy supply (between 1970 and 2005) has been reported in the Middle East and North African countries ${ }^{(25)}$, while the Chinese population is consuming more energy-dense food than previously ${ }^{(26)}$. In Brazil, dietary deficit is changing to dietary excess ${ }^{(27)}$. It was recently reported in Nepal that there has been a change from an agricultural staple-based foods diet to a modern processed-foods diet with higher total energy, total fat and sugar ${ }^{(28)}$.

\section{Macronutrient distribution}

Another salient observation is the changes in macronutrient intakes and macronutrient distribution (expressed as a percentage of total energy) over time. These changes followed the same pattern described as part of the nutrition transition in the NWP when rural and urban Africans were compared in a cross-sectional study ${ }^{(7,8)}$. The differences in intakes between rural and urban participants in 2005 were similar to the changes observed in both rural and urban participants over time (in 2010). The urban participants ate more in 2005 and 2010 than their rural counterparts. With a few exceptions, the changes included the following: (i) the percentage of energy contributed by total protein (except urban men), animal-derived protein, total fat (except urban women), saturated fat, monounsaturated fat and added sugar all increased significantly and reflected increased intakes of mainly meat, eggs and milk; (ii) the percentage of energy from plant protein decreased significantly in all groups; and (iii) the percentage of energy from total carbohydrate decreased significantly in rural participants despite the increase in added sugar (a carbohydrate which forms part of total carbohydrate), therefore this decrease probably reflects the decreased intake of starchy staple foods. Because these changes occurred mostly in rural participants it suggests that the diet followed by urban participants in 2005 (except for energy intake) was already modernized to a 'stable' level and that rural participants still had 'room to move' towards the energy distribution seen as a result of the nutrition transition. The change from a high-fibre-rich carbohydrate to a lower-carbohydrate diet with more sugar and sugar-sweetened beverages is also taking place in other countries ${ }^{(28-30)}$. In addition, a dramatic increase in meat consumption has been reported for China and Brazil $^{(31)}$.

An interesting finding is the significantly lower alcohol intake in rural and urban men in 2010 (both in grams and as a percentage of energy intake). A possible explanation could be that due to the nutrition transition, modernization and moving away from traditional home-brew methods, men consumed commercial beer rather than home-made beer. Commercial beer is more expensive and the higher price could have lowered its consumption.

Although we did not test for significance between men and women, it is interesting to note that women consumed more added sugar (in grams and as a percentage of energy; except for the rural women in 2005) than men. We previously showed ${ }^{(11)}$ that more women than men consumed sucrose-sweetened beverages in 2005 and 2010 and that the quantities were also larger. It could also be that the women consumed more sugar in their tea/coffee. 


\section{Micronutrient intakes: nutrient adequacy}

One would have hoped and expected that the fortification of maize meal and bread flour, two main staples in these populations, would correct micronutrient deficiencies of most people in SA. The present study, however, showed that intakes of the rural men and women in 2005 did not meet recommended values for most minerals and vitamins. The situation was better in the urban groups and improved over time in both rural and urban participants. Therefore, the nutrition transition resulted in improved, but still not optimal micronutrient intakes. The South African food composition tables incorporated new analytical data of the fortified staple foods. However, it was reported in 2012 ${ }^{(32)}$ that many of the samples of maize meal and white bread flour across SA that were analysed were insufficiently fortified. To assess the real extent of micronutrient deficiencies, suitable biological markers of the micronutrients will have to be measured in future. Nevertheless, the data showed that micronutrient needs in the PURE-NWP-SA participants were not met despite a high intake of energy, probably a result of more energy-dense but nutrient-poor foods chosen.

\section{Protective dietary effects}

The low intakes of dietary fibre and many of the antioxidant micronutrients are of concern. The low-fat, highfibre traditional diet of the indigenous people of Africa has been regarded as a major factor protecting them against $\mathrm{NCD}^{(2,33,34)}$. The nutrient intake changes observed here are indicative of a loss of this protection of the diet. Schutte and co-workers ${ }^{(14)}$ already reported that $24 \%$ of participants of the PURE-NWP-SA study who had normal blood pressure in 2005 presented with hypertension in 2010, while we showed the massive increased consumption of nutrient-poor sugar-containing beverages over this time ${ }^{(11)}$. Together with other changes during the epidemiological transition, the increase in NCD among the Africans in SA is not surprising.

\section{Limitations}

One of the major limitations of the PURE-NWP-SA study, namely the moderately high proportion of subjects lost to follow-up, of whom $30 \%$ died, was discussed previously $^{(11)}$. Those lost to follow-up were significantly younger, more often males, a higher percentage were HIV-infected, with lower BMI, waist circumference, HDLcholesterol and energy intake than those followed up. We suspect that the rest of the participants lost to follow-up, being younger and male, probably moved away to bigger cities where there are more opportunities for work.

\section{Conclusions}

It is concluded that the results of the current analysis of the PURE-NWP-SA nutrient data confirm a rapid nutrition transition in the NWP, characterized by large increases in total food energy without correcting fully for micronutrient deficiencies despite fortification of staple foods. Micronutrient intakes could even be lower because of insufficient fortification. Furthermore, the shift towards an animal food-based diet at the cost of a lower plant protein food intake could increase the risk of NCD. The data also confirmed that low dietary fibre and micronutrient intakes were prevalent, further contributing to a double burden of under- and overnutrition in this population.

\section{Acknowledgements}

Acknowledgements: The authors acknowledge the contribution of the late Professor Annamarie Kruger to the PURE South Africa study. They thank all supporting staff and participants in the PURE study; in particular, from PURE South Africa, the PURE-NWP-SA research team, fieldworkers and office staff in the Africa Unit for Transdisciplinary Health Research and the Centre of Excellence for Nutrition, Faculty of Health Sciences, North-West University, Potchefstroom, South Africa; and from PURE International, S. Yusuf and the PURE project office staff at the Population Health Research Institute, Hamilton Health Sciences and McMaster University, Ontario, Canada. Financial support: This work was supported by the South Africa Netherlands Research Program on Alternatives in Development; the South African National Research Foundation (GUN numbers 2069139 and FA2006040700010); North-West University, South Africa; the Population Health Research Institute, McMaster University, Canada; and the South African Medical Research Council. Funders were not involved in any aspect of the design, implementation, analysis, or interpretation and written account of the study. Conflict of interest: The authors declare that they have no competing interests. Authorship: E.W.-V. and H.H.V. were responsible for the conception and design, analysis and interpretation of the data and wrote the manuscript. S.L. made contributions to the design, analysis and interpretation of the data and critically revised the manuscript for important intellectual content. R.L. was responsible for the statistical analysis of the data and made contributions to the interpretation of the data and critically revised the manuscript for important intellectual content. Ethics of buman subject participation: The Ethics Committee of the North-West University approved the study (04M10 and NWU-0016-10-A1). The study complied with all applicable requirements of international regulations, including the Helsinki Declaration of 1975 (as revised in 2008). Permission for the study was also obtained from the NorthWest Provincial Department of Health, local government authorities, and tribal chiefs in the rural areas. Volunteers gave written informed consent. The participants were transported to and from the study sites in both the urban and rural areas. They received lunch on site after blood sampling was completed. The study is registered at ClinicalTrials.gov (ID NCT03225586, https://clinicaltrials.gov). 


\section{Supplementary material}

To view supplementary material for this article, please visit https://doi.org/10.1017/S1368980018001118

\section{References}

1. Pillay-van Wyk V, Msemburi W, Laubscher R et al. (2016) Mortality trends and differentials in South Africa from 1997 to 2012: second National Burden of Disease Study. Lancet Glob Health 4, e642-e653.

2. Vorster HH, Kruger A \& Margetts BM (2011) The nutrition transition in Africa: can it be steered into a more positive direction? Nutrients 3, 429-441.

3. Mayosi BM, Flisher AJ, Lalloo UG et al. (2009) The burden of non-communicable diseases in South Africa. Lancet $\mathbf{3 7 4}$, 934-947.

4. Nojilana B, Bradshaw D, Pillay-van Wyk V et al. (2016) Persistent burden from non-communicable diseases in South Africa needs strong action. S Afr Med J 106, 436-437.

5. Shisana O, Labadarios D, Rehle T et al. (2013) South African National Health and Nutrition Examination Survey (SANHANES-1), pp. 135-144. Cape Town: HSRC Press.

6. Norman R, Bradshaw D, Schneider M et al. (2007) A comparative risk assessment for South Africa in 2000: towards promoting health and preventing disease. S Afr Med J 97, 637-641.

7. Vorster H, Venter C, Kruger H et al. (2000) The impact of urbanization on physical, physiological and mental health of Africans in the North West Province of South Africa: the THUSA study. S Afr J Sci 96, 505-514.

8. MacIntyre U, Kruger H, Venter C et al. (2002) Dietary intakes of an African population in different stages of transition in the North West Province, South Africa: the THUSA study. Nutr Res 22, 239-256.

9. Serfontein M, Venter C, Kruger A et al. (2010) Alcohol intake and micronutrient density in a population in transition: the Transition and Health during Urbanisation in South Africa (THUSA) study. S Afr J Clin Nutr 23, 22-28.

10. MacIntyre U, Venter C, Kruger A et al. (2012) Measuring micronutrient intakes at different levels of sugar consumption in a population in transition: the Transition and Health during Urbanisation in South Africa (THUSA) study. $S$ Afr J Clin Nutr 25, 122-130.

11. Vorster HH, Kruger A, Wentzel-Viljoen E et al. (2014) Added sugar intake in South Africa: findings from the Adult Prospective Urban and Rural Epidemiology cohort study. Am J Clin Nutr 99, 1479-1486.

12. South African Department of Health (2013) Foodstuffs, Cosmetics and Disinfectants Act, 1972 (Act No. 54 of 1972). Regulations Relating to the Reduction of Sodium in Certain Foodstuffs and Related Matters. Pretoria: Government Gazette, Republic of South Africa; available at http://www. greengazette.co.za/.../index-for-20171006-national-gazetteno-41164

13. Corsi DJ, Subramanian S, Chow CK et al. (2013) Prospective Urban Rural Epidemiology (PURE) study: baseline characteristics of the household sample and comparative analyses with national data in 17 countries. Am Heart J 166, 636-646.e634

14. Schutte AE, Schutte R, Huisman HW et al. (2012) Are behavioural risk factors to be blamed for the conversion from optimal blood pressure to hypertensive status in Black South Africans? A 5-year prospective study. Int J Epidemiol 41, 1114-1123.
15. Sotunde O, Kruger H, Wright HH et al. (2015) Lean mass appears to be more strongly associated with bone health than fat mass in urban black South African women. $J$ Nutr Health Aging 19, 628-636.

16. MacIntyre U, Venter C \& Vorster H (2001) A culturesensitive quantitative food frequency questionnaire used in an African population: 1. Development and reproducibility. Public Health Nutr 4, 53-62.

17. MacIntyre U, Venter C, Vorster H et al. (2001) A combination of statistical methods for the analysis of the relative validation data of the quantitative food frequency questionnaire used in the THUSA study. Public Health Nutr 4, 45-51.

18. Wentzel-Viljoen E, Laubscher R \& Kruger A (2011) Using different approaches to assess the reproducibility of a culturally sensitive quantified food frequency questionnaire. $S$ Afr J Clin Nutr 24, 143-148.

19. Venter C, MacIntyre U \& Vorster H (2000) The development and testing of a food portion photograph book for use in an African population. J Hum Nutr Diet 13, 205-218.

20. Langenhoven M, Conradie P, Wolmarans P et al. (1991) MRC Food Quantities Manual. Cape Town: South African Medical Research Council.

21. Wolmarans P, Danster N, Dalton A et al. (2010) Condensed Food Composition Tables for South Africa. Cape Town: South African Medical Research Council.

22. Otten JL, Hellwig JP and Meyers LD, (editors) (2006) Dietary Reference Intakes: The Essential Guide to Nutrient Requirements. Washington, DC: The National Academies Press.

23. Nishida C, Uauy R, Kumanyika S et al. (2004) The joint WHO/FAO expert consultation on diet, nutrition and the prevention of chronic diseases: process, product and policy implications. Public Health Nutr 7, 245-250.

24. Vorster HH, Badham J \& Venter C (2013) An introduction to the revised food-based dietary guidelines for South Africa. $S$ Afr J Clin Nutr 26, 3 Suppl., S5-S12.

25. Sibai AA, Nasreddine L, Mokdad AH et al. (2010) Nutrition transition and cardiovascular disease risk factors in Middle East and North Africa countries: reviewing the evidence. Ann Nutr Metab 57, 193-203.

26. Popkin BM, Lu B, Zhai F et al. (2002) Understanding the nutrition transition: measuring rapid dietary changes in transitional countries. Public Health Nutr 5, 947-953.

27. Monteiro CA, Mondini L, de Souza AL et al. (1995) The nutrition transition in Brazil. Eur J Clin Nutr 49, 105-113.

28. Subedi YP, Marais D \& Newlands D (2017) Where is Nepal in the nutrition transition? Asia Pac J Clin Nutr 26, 358-367.

29. Malik VS, Walter CW \& Frank BH (2013) Global obesity: trends, risk factors and policy implications. Nat Rev Endocrinol 9, 13-27.

30. Mattei J, Malik V, Wedick NM et al. (2015) Reducing the global burden of type 2 diabetes by improving the quality of staple foods: the Global Nutrition and Epidemiologic Transition Initiative. Global Health 11, 23-23.

31. Vranken L, Avermaete T, Petalios D et al. (2014) Curbing global meat consumption: emerging evidence of a second nutrition transition. Environ Sci Policy 39, 95-106.

32. Yusufali R, Sunley N, de Hoop M et al. (2012) Flour fortification in South Africa: post-implementation survey of micronutrient levels at point of retail. Food Nutr Bull 33, 4 Suppl., S321-S329.

33. Walker A (1994) Highlights of nutrition and health/ill-health research in Africa: how my interest started and developed. $S$ Afr Med J Suppl., 2-4.

34. Popkin BM (2002) An overview on the nutrition transition and its health implications: the Bellagio meeting. Public Health Nutr 5, 93-103. 